\title{
"What Can I Do to Help Myself?" Somatic Individuality and Contemporary Hormonal Bodies
}

\section{Celia Roberts}

\begin{abstract}
Today health consumers and citizens are repeatedly asked to actively manage their own bodies and those of their families in order to maximize health outcomes. This contemporary demand can be theorized as a form of somatic individualisation: a subjectification process establishing new and ever-closer relations between bodies and selves. Somatic individuality, according to Novas and Rose (2000), involves citizens and health consumers in ever-increasing levels of responsibility for bodily care and consequent practices of prudence and caution about physical futures. This paper critically examines the concept of somatic individuality, asking both how these forms of "responsibilisation" are intertwined with normative gendering processes, and if there is a disjuncture between rhetorics of responsibility and patients' experiences in medical clinics. Two case studies of contemporary hormonal bodies are analysed: discourses describing the effects of endocrine disrupting chemicals; and discourses of menopausal women's use of hormone replacement therapy.
\end{abstract}

Keywords: individualisation, responsibility, sex hormones

In a glossy lemon and mauve leaflet produced by the National Health Service (NHS), British women are offered "the facts about the menopause, HRT [hormone replacement therapy] and osteoporosis" (Health Promotion England, 2000). After eight pages of facts, a new heading reads "What can I do to help myself?" The text answering this question is initially reassuring, stating that
"[S] ome women have little or no trouble with the menopause". Women are told not to assume it's going to be awful, and to remember that every woman has a different experience. "Even if you do have problems", it suggests, "there's a lot you can do to help yourself". A list is provided: "be prepared; eat a healthy, balanced diet, avoid smoking, stay active, use a vaginal lubricant, consider Hor- 
mone Replacement Therapy", and finally (no verb included) "alternative therapies" (Health Promotion England, 2000: 9). No mention is made here of doctors, and science only comes in to cast doubt on alternative therapies: "Some women find that alternative or complementary therapies like aromatherapy can help ease their symptoms and make them feel more relaxed. However, there is no scientific agreement on whether these treatments actually work" (Health Promotion England, 2000: 9). According to the NHS, then, British women can "help themselves" to cope with their unique experiences of menopause in apparently simple, practical and often non-medical ways.

The question "What can I do to help myself?" lies at the heart of contemporary British health policy: the Blair government's recent White Paper on health, "Choosing Health: Making healthier choices easier" (2004), employs a rhetoric of liberal self-help and autonomy. Cutting the apron strings of "the Nanny state", British citizens are figured in this document as people who can and should make their own choices about health and the prevention of illness. As Tony Blair clarifies in his introductory preface, within this framework "the Government cannot - and should not - pretend it can 'make' the population healthy. But it can - and should - support people in making better choices for their health and the health of their families" (Blair, 2004: 3 ). As indicated throughout the document, this means teaching citizens how to seek information and to act upon it in order to consume health messages, products and services.

This article investigates the parameters, dynamics and implications of the question "What can I do to help myself?" In particular, I ask whether the concept of "somatic individuality" - a term proposed by British sociologists Carlos Novas and Nikolas Rose (Novas and Rose, 2000; Rose and Novas, 2005) provides a useful framework to investigate contemporary patients' and citizens' experiences of biomedicine in the U.K and other developed nations with substantial state healthcare systems (Australia and Scandinavian countries, for example). The paper takes sex hormones and hormonal bodies as its central foci, suggesting that rather than remaining within the technical realms of biomedicine and technoscience, hormonal bodies are increasingly becoming something that citizens and patients are asked to do something about, in order to "help themselves". This is demonstrated through an analysis of two contemporary discourses: those concerning hormone replacement therapy (HRT), the standard biomedical treatment for problems associated with the menopause; and those describing the effects of environmental estrogens or endocrine-disrupting chemicals.

My analysis of these case studies produces two linked strands of argumentation. Firstly, I claim that theorising new responsibilities relating to the governance of the body requires attending both to the sexed nature of human bodies and to the deeply gendered histories of caring for bodies that constitute western cultures. ${ }^{1}$ Secondly, I develop a point mentioned only briefly by Novas and Rose (2000: 488): that somatic individuality is unevenly distributed across different material-discursive fields of medicine. Analysing HRT discourses, I find that menopausal women are only some- 
times subjectified as somatic individuals. Woven together, these two strands of argumentation problematise the notion of somatic individuality, demonstrating that it is, at best, unevenly distributed across different people and fields and, at worst, open to co-option by capitalist biomedical discourses in the pursuit of compliant consumer populations.

\section{Somatic Individuality and "Responsibilisation"}

In contemporary neo-liberal societies, it has been argued, life events are understood as risks that could and should be ascertained and managed (O'Malley 2000; Ericson, Barry and Doyle, 2000). In these societies, responsibility for risks is increasingly devolved to individuals: risks become something that should be taken (an entrepreneurial attitude towards life is rewarded), but also prudently reflected upon. As Pat O'Malley (2000: 465) argues in an article on contract law and uncertainty, "the prudent subjects of neo-liberalism should practise and sustain their autonomy by assembling information, materials and practices together into a personalized strategy that identifies and minimizes their exposure to harm". In the arena of health, this means a move away from a welfare state approach in which the economic consequences of ill health are shared across a whole society, towards a model, as Blair puts it in the quote above, of "supporting" individuals to become responsible for their own health risks. As Ericson, Barry and Doyle (2000) suggest in their analysis of the private insurance industry, this model of individual responsibility ultimately assumes that life events (ill health or accidents, for exam- ple) are the result of poor life management. The risks covered by insurance, consequently, are no longer mutualised but "unpooled" or borne only by specific market "segments".

Novas and Rose's (2000) concept of somatic individuality describes the contemporary "responsibilised" subject within neo-liberal states. It elaborates a Foucauldian argument about the modern embodied self and builds on American anthropologist Paul Rabinow's (1992) notion of "biosociality", itself a development of Foucault's "biopower" (Foucault, 1987: 140). Investigating medical genetic discourses, Novas and Rose suggest that contemporary biomedicine configures western subjects as responsible and active managers of our own embodied lives. This configuration within medical genetics is, they argue, part of "a wider mutation in personhood that we call 'somatic individuality' in which new and direct relations are established between the body and the self" (Novas and Rose, 2000: 487). In these new forms of personhood, human individuality is thought about and acted upon along bodily lines, in multiple arenas ranging from medical genetics through to feminist philosophy (Novas and Rose, 2000: 491). In relation to biomedicine, this move is one in which technical forms of knowledge about bodies move "from the esoteric discourse of science to the lay expertise of citizens" (Novas and Rose, 2000: 488). In the field of genetics, Novas and Rose propose, for example, that citizens/patients are not passive victims of "geneticisation" (as has been argued by Dreyfuss and Nelkin [1992] and Lippman [1992]) but rather actively engage with genetic discourses, thus producing new subjectivities. These 
new engagements or subjectications, they argue, are characterised by practices of choice, enterprise, and self-actualisation in which people understood to be "at genetic risk" become responsible for managing their genetic inheritance and hence their outcomes of their lives (Novas and Rose, 2000: 505; Rose and Novas, 2005).

This focus on individual responsibility and the formulation of personal life strategies is reflected in British health policy documents, as mentioned above. Other social research on health - including Jackie Stacey's (2000) discussion of “self-health” books, Ilpo Helén's (2004) analysis of prenatal testing and Silja Samerski's (2005) and Bernhard Wieser, Sandra Karner and Wilhelm Berger's (2005) studies of genetic counselling in pregnancy - also corroborates this view. Stacey, for example, demonstrates that complementary therapy discourses encourage health consumers to adopt new life strategies and to make interventions at physical, psychological and spiritual levels in order to re-establish lost harmonies of body, mind and spirit. Even these discourses, often set against western biomedicine, produce forms of individual responsibility for health and illness that are "new", at least within an Anglo-British context. In the context of pregnancy in northern Europe, Helén (Finland), Samerski (Germany) and Wieser, Karner and Berger (Austria) all suggest that women are increasingly asked to take ethical responsibility for complex decisions about prenatal testing and possible abortion. This responsibility fills an information gap between scientific knowledge about population risks and the difficulty of predicting risks for any individual (see also Griffiths,
Green and Bendelow 2006). The process of information-giving within genetic counselling sessions cannot, Samerski (2005) argues, provide women with a meaningful basis on which to make a judgment about a pregnancy because the information provided can never be about their personal risk. The gap between information (knowledge about the statistical risks for groups of women and kinds of pregnancies) and ethical decision-making (what to do about this particular pregnancy), Helén (2004: 37) shows, is filled by a demand that individual pregnant women make decisions for themselves. Framing this demand as patient empowerment, he argues, clinicians withdraw to a position in which they are only responsible for technical, rather than ethical matters. In the face of clinicians' inability to provide individually-specific information, then, each pregnant woman becomes "a subject of choice.... [who] has an opportunity to make a choice and is also compelled to do so" (Helén, 2004: 38). Citing Foucault (1985: 25-32) and following Novas' and Rose's lead, Helén suggests that such compulsion "requires practices of the self [...] the pregnant woman must analyse and work through her aspirations and feelings related to having a child and, indeed, her own life as a whole" (Helén, 2004: 38). She must, in other words, become responsible for answering the question, "What can I do to help myself?"

It is no accident that this shifting of responsibilities from medical professionals to patients - what Helén (2004: 40) calls "responsibilization" - takes place alongside steadily increasing levels of biomedical and technoscientific uncertainty about bodies, life and 
health. As biomedicine and technoscience come to know more about the complexities of life itself - about genes, hormones, multiple chemical interactions, environmental influences, psychological factors - it becomes more and more evident that "biology" is ultimately ungovernable in any particular instance. This, as Helén argues, is why there is so much contemporary talk of risk and risk management: "The uncertainties of life", he suggests, "become conceivable today risks pertaining to environments, populations or individuals" (Helén, 2004: 30-1). The requirement for individuals to undertake subjectivity-crafting decisions and actions to deal with these risks constitutes a core element of contemporary bio-power.

Like those surrounding genetics and reproduction, hormonal discourses are also full of uncertainties and the consequent shifting of responsibilities onto patients/consumers. In the case of endocrine-disrupting chemicals, as I shall show, consumers are asked to manage hormonal bodies in the face of serious gaps in technoscientific and biomedical knowledge. In the case of HRT - also an area of great biomedical uncertainty - a similar move is made but with an important twist: whilst women are given responsibility for decision-making in patient leaflets and self-help books, in clinical encounters they are often denied opportunities to make choices about HRT. This disjuncture across different material-discursive fields raises a question that is relevant across all areas of biomedicine, namely, "What are the material-discursive consequences of discourses of consumer choice and active engagement in the face of biomedical and technoscientific uncertainties?"
Rather than patients who can engage actively with biomedical professionals and thus materially take responsibility for their own bodies and lives, do these discourses ultimately produce more willing consumers of biomedical services and products (like prenatal testing or HRT)? If so, what are the implications for theorising patients/consumers as somatic individuals? ${ }^{2}$

\section{Case Study One Gendered Responsibilities: Managing the Effects of Endocrine- Disrupting Chemicals}

\begin{abstract}
The chemicals that have ushered in the modern industrial age are literally everywhere - in pesticides applied in everincreasing quantities to food crops, in plastic microwavable containers, in dental amalgams, in the resins that coat the inside of tin cans. For decades, such substances have generally been regarded as safe at low exposures. But new evidence suggests that relatively low levels of industrial chemicals may mimic or obstruct hormonal activity with potentially devastating long-term effects that range from cancer and reproductive abnormalities to cognitive dysfunctions like Attention Deficit Disorder (inside front cover, Hormonal Chaos).
\end{abstract}

In his book, Hormonal Chaos: The Scientific and Social Origins of the Environmental Endocrine Hypothesis, environmental policy expert Sheldon Krimsky (2000) describes the development of what he calls "the environmental endocrine hypothesis", the contention that our contemporary environments are thoroughly saturated with potentially toxic chemicals affecting hormonal systems. Stemming from both industrial and agricultural sources, these chemi- 
cals "have the capacity to mimic or obstruct hormone function - not simply disrupting the endocrine system like foreign matter in watchworks, but fooling it into accepting new instructions that distort the normal development of the organism" (Krimsky, 2000: 2). These disruptions are linked in both human and non-human animals to increased rates of reproductive abnormalities, cancers and immune dysfunction. The chemicals included in this category are familiar: the pesticide DDT (now widely banned); organochlorides (also used in pesticides); PCBs (polybrominated biphenyls, found in plastics); and bisphenol A (the laqueur coating used in food cans). As well as these chemicals of "the modern industrial age", our environments are also increasingly polluted by hormones deriving from medications. British rivers, for example, contain detectable levels of estrogens excreted by users of the contraceptive pill (Krimsky, 2000: 66). Like their industrial and agricultural counterparts, these environmental estrogens are thought to have serious implications for the sexual development of fish and potentially also of humans and other animals consuming these fish. As Krimsky argues, the combination of all these chemicals creates currently incalculable risks for humans and other animals. Whilst exposure to all of these chemicals in tiny amounts has always been understood as safe, the combined and cumulative affects of lifetime exposures are extremely difficult to study and remain contested (see also Sharpe and Irvine, 2004).

The uncertain yet potentially serious risks posed by endocrine-disrupting chemicals has led in recent years to a plethora of discourses describing their impacts, contesting their industrial use and promoting strategies for containing their effects. These discourses are produced by scientists, governments, journalists and other writers, environmental groups and corporations. Here I focus on discourses providing information and advice to consumers about the effects of endocrine-disrupting chemicals from environmental organisations, popular books and media reports.

Environmental organisations such as Greenpeace, Friends of the Earth and the World Wildlife Fund, have initiated campaigns based on evidence provided by laboratory and observational studies of endocrine disruptors. These campaigns aim to produce awareness and a sense of responsibility in members of the public, as well as to increase pressure on governments and corporations to regulate the production, use and disposal of endocrine disruptors. In these campaigns, consumers are asked to manage their own and others' bodies in order to minimize the diffuse yet serious threat of endocrine disruption. This responsibilisation for new hormonal bodies is, I suggest, often gendered.

The World Wildlife Fund (WWF) campaign is part of its broader "Toxics Programme" (WWF, 2006) and has multiple facets, including web pages, reports, position papers, publicity and educational stunts, pamphlets and posters. One of the campaign posters, published on the web, in newspapers and in women's lifestyle magazines, highlights the gendering of new responsibilities for dealing with hormonal bodies. Alongside a red-orange, luminescent close-up of a well-developed human foetus, the poster incites us to "take action for a living planet" by trying to replicate the womb of our 
grandmothers. "The womb should be the safest place on earth", the text claims, "but today our bodies are contaminated with over 300 man-made chemicals, to which our great grandparents were never exposed" (WWF, 2002). The poster evokes the womb as a place of unreasonable danger for a peaceful foetus, figuring an ideal of natural purity and a chemical-free historical past. Invoking a biologistic kinship discourse, mothers and potential mothers are encouraged to take action so that "so that the only thing we pass on to our children is our genes".

This use of pregnant women's bodies to represent that which is threatened by endocrine disruptors is widespread. Greenpeace campaigns, for example, figure women both as toxic conduits to future children and as contaminated "nature". On their webpage, "Poisoning the Unborn" (Greenpeace, 2005), a beautiful, naked pregnant woman lies along the bottom of a spherical under-water window, looking out into a blue ocean, protectively cradling her belly. The front cover of the report "Human Impacts of Man-made Chemicals" (Greenpeace, 2003), also uses an image of a naked pregnant woman; this time standing. Cropped at the neck and hips, the woman is reduced to her enormous belly and breasts cradled, again, by her protective arms. Images further down the "Poisoning the Unborn" webpage include a girl playing with plastic toys and a man vacuuming a bedroom: he is not cleaning, however, but "collecting dust samples from homes to test for the amount of toxic chemicals" (Greenpeace, 2005: 2). The Friends of the Earth's "Safer Chemicals" campaign uses similarly gendered images. Their colourful fold-out "parent's guide" to "chemicals in the home" depicts women engaging in dangerous domestic practices (cleaning, spraying air fresheners, putting on makeup, breastfeeding and being pregnant) and a male Professor giving expert advice (Friends of the Earth and the National Childbirth Trust, undated). All these campaigns address women as mothers, home-makers and child-carers and depict ordinary domestic actions and women's reproductive bodies as dangerous to others. Men - if present at all - represent scientific expertise.

These campaigns and other discourses around environmental estrogens, I suggest, (re)cite conventional versions of sex/gender and caring relationships through figuring women as responsible for their own and others' hormonal bodies. Popular books and media articles also advise women to modify their caring behaviours in dealing with the threats of endocrine disruptors. D. Lindsey Berkson's (2000) Hormone Deception addresses a female readership, advising "how to protect yourself and your family" through the careful choice of everyday products like food and cleaning products. She provides a "room-by-room tour" of "the home", describing the potential hazards associated with each kind of space. As in the environmental campaigns, women's bodies are also figured as conduits of toxicity. Berkson illustrates and describes pregnant women unwittingly sharing endocrine-disrupting chemicals with their foetuses: "Women carry and nurture within their bodies our future generations - the innocent foetuses who are the most vulnerable of all when exposed to hormone disruptors. The substances that a mother has been exposed to 
throughout her life, and some of what she takes in during her pregnancy, become the legacy of the next generation. So the womb is really the first environment with which we should be concerned" (Berkson, 2000: 81).

Berkson's focus on everyday products and women's bodies is shared by articles in the media: articles in a range of British newspapers - The Daily Mail, The Times, The Independent, Daily Telegraph and The Guardian - discuss endocrine disruptors along these lines. ${ }^{3}$ The Guardian substantially developed this theme in 2004 in a series of articles and supplements discussing our "ChemicalWorld", beginning with a story about pesticideuse in Spanish salad farms. Excerpted from a book by Felicity Lawrence, this story informed British consumers that so-called "pre-washed" salad is laced with high levels of chlorine and pesticides and certainly should at least be washed, if consumed at all (Lawrence, 2004). Over the following three weeks, The Guardian published three full-colour magazines addressing chemicals in food, in the home and in beauty products. Readers were provided with detailed accounts of the "average" person's exposure to endocrine-disrupting chemicals in everyday life. In one article, ordinary products that one might put on the pure skin of a baby were explained to contain "a cocktail of chemicals” (Atkins, 2004: 20). Specifically addressed itself to mothers (one part of the article is called "When you're pregnant") the article, like the WWF campaign discussed above, compares contemporary wombs with those of "our [great] grandmothers": "From the day they are conceived, our children are exposed to a soup of chemicals, most of which would not have existed when our grandmothers were in the womb" (V. Howard, cited in Atkins, 2004: 22).

As would be expected following Helén's argument about uncertainty and responsibilisation, all of this unnerving information about endocrine disruptors is accompanied by recommendations for "decision and action" by responsible individuals. These advocate consumer choice, limiting the use of chemicals in the home, and returning to the practices of "our grandmothers" (using lemon juice as a cosmetic, for example). Consumers are advised to not wash their hair too much, not to wear too much sunscreen, to tone their faces with cold water rather than skin toner, not to use cling-film, to trim off the fat from their meat, to buy organic fruit, not to let their babies use old plastic bottles and plates or chew plastic toys, to use olive oil on their babies' bottoms and to wash their hands after being outside. Like those targeted by environmental organisations and popular books, these areas of action are distinctively feminine, focussing on the care of bodies and babies and responsibilities for shopping and food preparation. ${ }^{4}$ The answers provided to the question "What can I do to help myself", in other words, configure a female interrogator. It is women who are encouraged to manage the hormonal bodies at risk from external forces. Somatic individuality is certainly being produced here, but the attendant responsibilities are not evenly distributed. In relation to endocrine disruption, somatic individuality is intimately linked with gendering processes and gendered practices of responsibility and care for others. 


\section{Case Study Two \\ Hormone Replacement Therapy and Uneven Distributions of Responsibility}

My second case study illustrates the uneven distribution of responsibilities attached to somatic subjectifications in terms of discursive-material fields. In menopause and HRT discourses, women are asked to manage another set of risks in relation to hormonal bodies: those pertaining to using sex hormones as a treatment for menopausal symptoms and to prevent illnesses associated with aging. Analysing a range of contemporary HRT-related patient literatures and a set of empirical studies of clinical encounters around the menopause highlights a serious disjuncture between the assignment of responsibility in patient leaflets and the experiences of patients in menopause clinics. This disjuncture in turn raises questions about the meaning and function of somatic individuality within contemporary biomedicine.

\section{Menopausal Women in Patient Literature}

Helén (2004) argues that in contemporary biomedical discourse, information is supposed to mediate between population risks and individual risks. Discussing the example of prenatal testing, he writes: "The crucial transmitting element in this assemblage is information. Information is supposed to translate group, clinical or technical risks into a form that enables pregnant women to assess the risks and decide whether or not they should undertake the procedures of screening or testing" (Helén,
2004: 35). Information, in other words, is what biomedicine offers to consumers or patients so that they can make decisions about their bodies. This is certainly true for hormonal bodies. In the case of endocrine disruptors, female consumers are hailed with attentiongrabbing images and advice about how to deal with their own and their children's bodies in the face of great uncertainty about the effects of these chemicals. In the more medical arena of the menopause, information is offered in a more sober form, in leaflets promising women "the facts" needed to make decisions about body management and HRT-taking and popular books promoting informed lifestyle and medical choices. $^{5}$

In the NHS leaflet described in the opening of this article, the menopausal woman is told that HRT "is something you should consider carefully and discuss with your doctor" (Health Promotion England, 2000: 17). She is advised to "find out all the information you can about HRT" and is given a long list of questions to pose in discussions with her doctor, including, "Could HRT help me?", "Does it cost anything?" and "Are there other options for treating my symptoms?" (Health Promotion England, 2000: 17). The responsibility for making decisions about HRT, however, remains with the patient/reader. "You will need to weigh the benefits to yourself against the possible risks", the leaflet suggests: "Much will depend on your medical history, symptoms and state of health" (Health Promotion England, 2000: 17). Like Helén's pregnant woman, the menopausal woman is supposed to bring the "facts" presented in this leaflet to bear on her own life and body and to 
decide whether "HRT is right for you" (Health Promotion England, 2000: 14). ${ }^{6}$

Since the publication of this leaflet in 2000 , the level of biomedical uncertainty surrounding HRT has substantially increased. In 2002, the world's largest clinical trial of HRT was prematurely terminated because early data showed that rather than preventing coronary heart disease as promised, HRT appeared to be increasing women's risk of this condition, as well as stroke and pulmonary embolism (Writing Group for the Women's Health Initiative Randomized Controlled Trial, 2002). The women taking HRT in this study were also found to incur a $26 \%$ increased risk of invasive breast cancer. In the scientists' press release, women are advised to have a "serious talk with their doctor" about continuing with HRT (National Institutes of Health, 2002).

The termination of this trial was widely publicised in the British media, causing significant concern amongst HRT consumers. ${ }^{7}$ In response, pharmaceutical companies and clinics distributed more "facts" about HRT. A leaflet produced by HRT manufacturer Novo Nordisk provides an example. Although entitled "Choosing HRT: weighing up the facts", this scientific-looking glossy page of A4 makes only a weak assertion that it might be helpful to women, stating that it "has been produced to help you decide whether HRT is right for you. Weighing up the associated benefits and potential risks may help you to make that decision" (Novo Nordisk, undated; emphasis added). Mentioning the " associated benefits" first, the leaflet makes strong claims without any statistical evidence, listing both the relief of menopausal symptoms and the prevention of osteoporosis and coronary heart disease as outcomes of taking HRT. Moving on to the "potential risks", however, the leaflet enters the realm of statistics. "You may be concerned about the possibility of developing breast cancer while on HRT", it asserts (and if the reader has seen with the media coverage of the Women's Health Initiative trial this is indeed highly likely). Stating the logical truism that "[A]ll women are at risk of developing breast cancer, whether they are on HRT or not", the leaflet then runs its deceptively simple argument. Using a bar graph, the reader is shown how the number of women "likely to be diagnosed with breast cancer" increases with the length of time they have taken HRT. This statistical fact is explained as if it refers to a specific (and very small) number of women: "For women aged fifty who start HRT only an extra 6 in 1,000 cases will be diagnosed after 10 years". We are not told how many women this actually refers to in any one year, or which women these might be. The leaflet also provides statistics on "the risk of developing deep vein thrombosis, 3 in 10,000 whilst taking HRT, in comparison to 1 in 10,000 for women not taking HRT”. Again, no information is given about the characteristics of these women or the significance or meaning of these numbers. These statistics are described in small print at the bottom of the page as "some of the necessary facts required to help you make a wellinformed decision about HRT", but without at least a working knowledge of statistics or epidemiology, they are meaningless. Their role, arguably, is not to "give the facts", but rather to demonstrate that the risks associated with HRT are minimal and only "potential”, in con- 
trast to the benefits, which are "wide ranging" (no graphs are provided to demonstrate these). In order to elaborate what these figures mean for her personally, the menopausal woman is referred to her general practitioner for further discussion.

In both leaflets, women are given "facts" and encouraged to seek help with decision-making about HRT through initiating discussions with general practitioners. The somatic individual represented here is someone who engages actively with biomedical knowledge and is able to openly question her doctor about the effects of medication, so that she can make an informed decision. Similar representations are found in popular self-help books on the menopause such as Jenni Murray's (2001) Is It Me, Or Is It Hot In Here? In a discourse analysis of four such books available in England, Lyons and Griffiths found that "the medical profession was constructed as primary source of expertise on menopause and women's bodies, although responsibility for the 'management' of menopause as a chronic condition lay solely within individual women" (Lyons and Griffiths, 2003: 1629). Although "menopause, women and their bodies" are presented as "too complex for them (or even the medical profession) to understand", women are constantly told that "gaining information is the best way.... to help themselves at midlife" (Lyons and Griffiths, 2003: 1639-1640). This information, however, in two of the books studied, "is only useful insofar as it makes women better equipped to talk to their doctors" (Lyons and Griffiths, 2003: 1640). Responsibility for managing the menopause, as in the leaflets analysed above, focuses on acquiring medi- cal knowledge and assistance (Lyons and Griffiths, 2003: 1641). This is a powerful vision of contemporary patienthood, one that certainly challenges older figurations of acquiescent, silent "victims" (see also Murtagh and Hepworth, 2003a). But how is this somatic individuality materially enacted in clinical spaces associated with the menopause and HRT-prescription? ${ }^{8}$

\section{Menopausal Women in the Clinic}

A series of recent empirical studies indicates that somatic individuality is extremely difficult to enact in menopauserelated doctor-patient encounters. Although there is evidence that both patients and doctors espouse discourses of patient empowerment and informed choice (Griffiths, 1999; Griffiths et al., 2005; Griffiths et al., 2006; Hunter, O'Dea and Britten, 1997; Murtagh and Hepworth 2003a and b), this research indicates that HRT is most often prescribed without detailed attention to health risks, alternatives or even patient preferences. Importantly, these studies come from the UK, Scandinavia and Australia, all countries that promote the idea of the informed health consumer. As a group they have varied aims and intended audiences and utilise a variety of social scientific research methods, including interviews, observations, simulations and ethnography. My discussion here is a kind of qualitative meta-analysis: a comparison and compilation of results and arguments about the clinical encounters they describe.

One of the most significant findings of these studies is the difficulties doctors experience in communicating risk information to patients. In their analysis of 
109 audio-taped consultations, British researchers Frances Griffiths, Eileen Green and Maria Tsouroufli (2005) found that a range of clinicians (specialists, general practitioners and nurses) found communicating the uncertainties of medical evidence about HRT very difficult. Significantly, Griffiths et al. noted that uncertainties tended to be expressed only in discussions with patients who had already taken HRT and who were considering continuing or restarting after a break. In discussions with patients new to HRT, uncertainties remained unmentioned: clinicians told women that HRT was effective in the prevention of osteoporosis and in the treatment of menopausal symptoms. In such consultations, although "the intention seemed to be to provide information and explanation so that the woman could make her own decisions... the overall tenor of the consultations was in favour of the intervention" (Griffiths et al., 2005: 513).

This failure to discuss risk was not found in a comparable Swedish study. In an observational analysis of 20 consultations between menopausal women and five different physicians, Mikael Hoffman et al. (2005) found that the risks of taking HRT were discussed in 18 consultations. Importantly, however, in 8 of these the decision to prescribe HRT was made before the risk discussion was initiated or completed. Even more worryingly, in all 20 cases, HRT was prescribed whether or not the woman stated that she desired this (Hoffman et al., 2005: 5). Even women who explicitly refused to take the drug went home with a prescription and without having had an opportunity to discuss alternative approaches. Hoffman et al. found that phy- sicians focused strongly on the prevention qualities of HRT rather than its ability to alleviate menopausal symptoms and used the discussion of risk to "motivate the woman to follow the physician's decision rather than to help her participate in the decision-making process" (Hoffman et al., 2005: 1). HRT was discussed by all but one physician "without any comparison with other treatment strategies" and without reference to any but the most basic statistics on breast cancer risk (patients were given the absolute risk for the average woman without any risk factors, with or without HRT) (Hoffman et al., 2005: 9). Given that the participating physicians knew that this study was focusing on discussions of risk and that the consultations were being tape-recorded, it is safe to assume that they were trying their best: as the authors state, "these consultations constitute... possibly a best-case scenario for discussion of risks and benefits associated with HRT" (Hoffman et al., 2005: 8). Despite this, patients were barely able to express concerns and physicians did not indicate any ability to listen to these, if expressed.

The lack of space in which to discuss concerns was also evident in Griffiths et al.'s research and in another study based in south Wales. Here, Glyn Elwyn et al. (1999) simulated doctor/patient discussions of menopausal symptoms and the possibility of taking HRT (along with 2 other scenarios) with 39 junior doctors. The purpose of the study was to investigate doctors' skills in facilitating shared decision-making. The authors found that these doctors did not have such skills: they were unable to describe risks to patients and did not feel that they had the necessary information to do so. In 
focus-group discussions after the simulations, doctors "admitted that 'friendly persuasion' was their usual practice, justified on the grounds that the responsibility of being involved in decisions would lead to increased (and by implication unacceptable) anxiety in patients" (Elwyn et al., 1999: 756). Like the Swedish study, these data indicate that doctors make decisions about what treatment to offer before discussing risks. One participant stated: "You choose the data to help the patient make the decision you think they ought to make. I'm sure I do that" (Elwyn et al., 1999: 755). Another said, "if the doctor feels that one course of treatment is better than another course of treatment, then that should be strongly pressed home" (Elwyn et al., 1999: 755). The editorial of the issue of the British Medical Journal (BMJ) publishing this study (which contains a number of studies of patient involvement in decision-making) accordingly comments that "paternalism is endemic in the NHS" (Cleary, 1999: 719).

The BMJ's reference to paternalism brings issues of gender to the fore. The enactment of medical authority in these studies is traditionally gendered: even if the doctors in question are women, they nonetheless enact an authority that is coded as masculine in its reliance on technoscientific rationality and expertise. ${ }^{9}$ Empirical evidence shows that faced with such "paternalistic" authority, menopausal women struggle to articulate alternative knowledge claims or to pose potentially undermining questions. Interviewing 32 women about their expectations and experiences of clinical encounters around HRT, Flis Henwood et al. (2003) found that although about half the women actively sought information about the menopause outside of the clinical encounter (on the internet for example), all the women interviewed expressed "great concern about appearing to over-step the boundary between 'expert' and 'patient'" in clinical encounters (Henwood et al., 2003: 601; see also 598 and 604; and Hunter et al., 1997: 1546; Massé et al., 2001). Interpreting interviewees' comments on this topic, Henwood et al. suggest that " $[C]$ learly, there is a distinction to be made between informing oneself about one's specific health condition and treatments and being prepared, or feeling able, to disclose what one has found out to one's doctor" (Henwood et al., 2003: 602). In their accounts of clinical experiences, some interviewees told stories in which they "seem to have been denied their right to make 'an informed choice' about HRT" (Henwood et al., 2003: 602). These women were not told about side effects and reported feeling dismissed when raising concerns about risks. As in the Swedish case, women in this study also reported coming away from clinics with prescriptions they did not want and had no intention of using (Henwood et al., 2003: 603). Henwood et $a l$. conclude their paper by suggesting that the structures and relationships currently constituting British general practitioner clinics will not allow for the "natural or easy" emergence of the informed patient (Henwood et al., 2003: $605)$. This conclusion is affirmed by a commentary on the Griffiths et al. study, also published in the BMJ. Here clinician Sandra Tanenbaum (2005) suggests that clinicians' difficulties in discussing risks with patients may reflect structural problems in contemporary healthcare systems in which resources are scarce 
and doctors are encouraged to sort people quickly into categories suitable for particular treatments.

Australian research also demonstrates the failure of clinicians to engage women in active decision-making about HRT. In an interview-based study of general practitioners, Madeleine J. Murtagh and Julie Hepworth (2003a: 198-9; 2003b: 1646) found that decisions to prescribe HRT were made before meeting patients. Although doctors espoused views that their role was to assist women to make informed choices, some nonetheless spoke of "hammer[ing] HRT very hard" and believing that "the best way I can help them is to offer hormone replacement therapy" (Murtagh and Hepworth, 2003a: 198). These data suggest that there is a significant gap between doctors' espousal of an informed consumer model in relation to menopausal women and their discourses around their own practices of clinical consultation. Murtagh and Hepworth explain this gap through referring to doctors' adherence to a limited biomedical model of risk. "While general practitioners lay claims to enabling and empowering women and supporting women's choices", they argue,

[T] hey actually limit choice through constructing menopause solely within biomedicine. In other words, by producing knowledge exclusively within a biomedical paradigm, when a woman enters the doctor-patient consultation these limits are already in place. One way in which this discursive construction of practice related to menopause becomes maintained and reproduced is through medical practitioners' using a range of strategies which have the effect of marginalizing knowledge from without this paradigm. (Murtagh and
Hepworth, 2003b: 1646)

Ethnographic research also complicates the idealised story of women's involvement in complex decision-making represented in the menopause patient literature. In her study of five menopause clinics in Melbourne, Marilys Guillemin (2000a, 2000b) found that women were often surprised by the way in which they received prescriptions for HRT. These prescriptions seemed to come rapidly and without discussion. One interviewee reported: "I had gone to the menopause clinic wanting to get more information and I was virtually put straight on HRT, or after the first visit when I had tests and things. In a way I was a bit shocked because they seem to have taken it for granted that's what I wanted and didn't really tell me if there were any alternatives" (Guillemin 2000b: 19). Another woman, Guillemin writes, felt "bewildered following her second consultation at the clinic. She had initially gone there just for information, and she found herself walking out the door with medication that she was a little dubious of and facing the prospect of at least ten years of therapy" (Guillemin, 2000b: 462). These stories show the collapse of the space between informationgiving and prescription: Guillemin's interviewees go to clinics for information and receive diagnoses and prescriptions for long-term HRT.

Analysed as a set, these studies cast doubt on the existence of opportunities for women to engage in serious dialogue with clinicians around HRT-related decision-making. In each example, women have serious difficulties discussing their concerns with doctors and in many cases "walk away" from clinical encoun- 
ters with prescriptions for drugs they did not desire. It seems, then, that discourses describing women's active role in "choosing HRT" serve a different role than producing clinical spaces in which concerns around HRT can be seriously explored. These discourses do not translate into clinical practice (even in bestcase scenarios, according to Hoffman $e t$ $a l$. [2005]), but rather enact a certain instantiation of the HRT-taking woman future and present (that is, those who will take HRT and those who already do). These discourses, in other words, enact a feminine subject who, despite behaving responsibly by engaging in almost impossible conceptual work around her body, is usually both deprived of acting on this work and highly likely to receive a HRT prescription if she attends a medical clinic.

\section{The Anxieties and Responsibilities of Gendered Somatic Individuals}

Experiencing demands to make impossible decisions can create serious anxieties. This has been demonstrated in the case of pregnancy and genetic testing in the work of Rayna Rapp (1999) and Barbara Duden (1993) amongst others and in the case of cancer by Stacey (1997). Helén suggests that the production of pregnant women as "choosing and consenting individuals by the practices of high-tech reproductive medicine" creates significant "existential responsibilities" and resultant anxieties (Helén, 2004: 38 and 40). This is because the decisions they are asked to make are understood only in personal terms. "Much of the perplexity of the condition of somatic individuality", he argues, "and of the 'vital governmentality' that is emerging alongside it, originates from the fact that our liberal order does not articulate, either in terms of ethics or (etho)politics, any other form for living these anxieties than personal choice" (Helén, 2004: 45). Both of my case studies affirm this claim, suggesting that in the fields of endocrine disruption and HRT and the menopause, women are asked to become personally responsible for caring for hormonal bodies. These responsibilities, I have suggested above, are not elaborated in terms of collective politics or ethics but rather in terms of consumption, domesticity and maternity (in the case of endocrine disruptors) or in terms of a discourse of informed patienthood (in the case of HRT). But what happens to this argument about somatic individuality if it turns out that consumers do not actually have much choice in practice? Arguably, this contradiction could increase the anxiety suffered, as the patient experiences failure in trying to enact the active choosing subject promoted in the patient literature given to her in hospitals and clinics: going home with a prescription that you have specifically said you do not want is presumably at least discomforting. Although none address this issue directly, existing empirical studies provide indications of such anxieties. Guillemin (2000b) describes women feeling "bewildered" and "shocked" by their experiences in menopause clinics and Henwood et al. (2003: 601) report women's "great concern" and "fear" (Henwood et al. 2003: 598) about displaying potentially contestatory knowledge in clinical encounters. These feelings are linked to anxieties about managing clinical encounters: Henwood et al. describe one woman who "feels the 
need to protect the doctor from 'the informed patient' who she sees as exerting extra pressures on an already busy professional" (Henwood et al., 2003: 602) and quote others describing themselves as "having to be very careful" about disclosing their knowledge and considering such disclosures "very inappropriate" (Henwood et al., 2003: 602).

The disjuncture between representations of somatic individuality in patient literature and the experiences of women in menopause clinics indicates that social theorists should be cautious in describing the "responsibilisation" of contemporary medical consumers/patients. As Novas and Rose (2000: 488) acknowledge, somatic individuality "operates alongside, and intersects with other modes of personhood operative in diverse practices and locales". Investigating hormone discourses suggests that, rather than being socially pervasive, somatic individuality is unevenly distributed, both in relation to sex/gender (responsibilities for managing hormonal bodies fall unevenly on women in the case of endocrine-disrupting chemicals) and within different material-discursive fields of biomedicine (patient leaflets and clinical encounters in the case of HRT). Consequently, we need to ask whether discourses of somatic individuality, rather than seriously challenging traditional configurations, enact new subjectifications that ultimately do little to challenge old hierarchies between patients and doctors. Instead of providing a wider range of options or establishing new practices of bodily self-management, discourses of individualised patient responsibilisation may, at least in some arenas, work to produce willing and compliant consumers for medical services and/or pharmaceutical products. ${ }^{10}$ Although active, informed patienthood has a radical history (within the fields of HIV/AIDS and breast cancer, for example), contemporary somatic individualisations can be appropriated by biomedical and pharmaceutical discourses in seriously limited ways.

Understanding how this might work in practice, I suggest, involves incorporating sex/gender into theorisations of somatic individuality. Despite the conceptual strengths of their work, neither Helén nor Novas and Rose engage with sex/gender in the papers cited here. Even in speaking of prenatal testing - a form of biomedicine that takes place in and around pregnant women's bodies they fail to take into account the different ways in which women and men experience their bodies, lives and demands to make choices about these. ${ }^{11}$ For a woman, arguably, what Helén calls "her own life" (Helén, 2004: 39) is not her own in the same way that a male partner's or comparable male patient's body might be. Helén argues that the concept of "own-ness" (the sense of having "one's own life") relevant to biomedicine today operates at three levels: firstly, as something that defines the individual as a member of a population; secondly, as the transformation of objective findings into a "personal biology" with risks; and, thirdly as “a person's own life, in the sense of personal experiences, choices, ways of living and personality" (Helén, 2004: 44). Somatic individuality, he suggests, operates through all these levels, but is always about "the problematization of a person's relation to him- or herself" (Helén, 2004: 44). What is lost in this tripartite figuration, is the person's relation to individual others. 
Empirical studies of medical arenas in which men and women are treated as couples (pregnancy, IVF, prenatal testing and preimplantation genetic diagnosis) show that patients' responsibilities for their "own lives" and the lives of others are complex and taken very seriously (see, for example, Duden, 1993; Rapp, 1999; Throsby, 2004; Roberts and Franklin, 2005; Thompson, 2005; Locock and Alexander, 2005). Studies of IVF and prenatal testing show that these responsibilities are gendered (Rapp, 1999; Thompson, 2005; Locock and Alexander, 2005; Roberts, 2006). In Karen Throsby's (2004) study of couples who had experienced unsuccessful IVF treatment, for example, female interviewees reported taking significant responsibility for preparing for treatment cycles and often held themselves physically and sometimes also morally responsible for their failure. Their male partners, on the other hand, often avoided undertaking even the most basic forms of preparation (such as wearing loose underwear or avoiding hot baths) and rarely blamed themselves when treatment was unsuccessful (Throsby, 2004: 134-162; Throsby and Gill, 2004). Indeed in conversations with friends, some men attributed failure to their wives' bodies in order to avoid being viewed as infertile themselves (Throsby, 2004: 149-150). Throsby (2004: 160-1) argues that both men's and women's attributions indicate the limited nature of vocabularies and emotional scripts available for discussing reproduction, health, bodies and distress. They also demonstrate how women are discursively and materially produced as responsible for others: women interviewees talked of feeling guilty about the impact of IVF treatment failure on their male partners and their own mothers, as well as on their doctors. Male interviewees did not express these concerns. Throsby concludes that IVF patients take on responsibility around treatment failure in what she calls "profoundly gendered" ways (Throsby, 2004: 136).

Although lacking this direct sex-based comparison, hormonal discourses also demonstrate that responsibilisation is deeply intertwined with ongoing enactments of sex/gender. In environmental discourses around sex hormones, women are figured both as conduits for dangerous chemicals and as responsible caretakers for the bodies of others. Women engaging with doctors around HRT decision-making fail to enact a model of empowerment that challenges conventional representations of patients as passive (and therefore feminised) in the face of (masculine) medical authority. Both cases reproduce historicallyenduring material-semiotic enactments of femininity: as in IVF, women are materially and discursively positioned as not simply controlling their "own" lives, but as responsible for the lives of connected, or potentially connected, others. The allocation of responsibility for hormonal bodies, in other words, lines up with historically-significant patterns of gendering in relation to care, authority and embodiment.

\section{Conclusion}

The concepts of somatic individuality and responsibilisation have much to offer contemporary analyses of biomedical discourse. The production of new subjectivities and responsibilities focused around bodily practices and concerns is strongly borne out in hormonal 
discourses, as well as in those around reproduction and reproductive technologies. It has been argued here, however, that somatic individuality is produced within existing complex networks of subjectification that include (but are not limited to) enactments of sex/gender. Somatic individuality and responsibilisation are unevenly distributed across different fields of biomedicine and across different bodies. This needs to be more seriously taken into account in current sociological debates.

The uneven distribution of somatic individuality and responsibilisation highlights the limitations of new subjectifications as challenges to traditional networks of power in biomedicine. Although different to older enactments of passive, uninformed patients/consumers, somatic individuals can be enrolled into biomedical discourses, services and products in highly restrictive ways. As some work is beginning to show (Rose and Novas, 2005; Dumit, 2002), the rise of somatic individuality is historically entwined with the development of corporate biomedicine and bears identifiable marks of this, alongside indicators of its more radical or resistant histories. Arguably the difference between the two is the formers' focus on individuals' responses to the management of health risk. Although many forms of health activism (such as those around HIV/AIDS, breast cancer or disability) also attempt to produce active, knowledgeable consumers of biomedical services and products, they concurrently maintain a focus on patients as collectives (Callon \& Rabeharisoa, 2004; Epstein, 1996; Batt, 1996; Rapp, Heath \& Taussig, 2001). Within patient activism of this kind, patients act collectively to access biomedi- cal resources, to change drug-testing protocols, to generate and have a say in directing research funds, to improve patient care and to change public perceptions of particular diseases and patients. Although this may sometimes involve collaboration with the pharmaceutical industry or government (see, for example, Epstein, 1996; 2003; Batt, 1996; Bix, 1997), the aim is to create new forms of (somatic) subjectivity through collective activity.

A focus on individuals, in contrast, has less potential to disrupt existing formations of power. Although, like patient organizations, pharmaceutical companies and governments articulate discourses of informed patienthood and are willing provide "information" to patients/consumers/citizens, I have argued here that far less is done to create material-discursive spaces in which these new forms of subjectivity can be enacted within biomedicine. As the history of collective patient activism shows, facilitating such space would have more enduring consequences for challenging traditional enactments of patients/consumers/citizens and of doctors/producers/governments than simply providing information. Providing information and passing on ethical responsibility for decisions to patients/consumers/citizens is, in my view, neither an adequate nor ethical response to contemporary biomedical uncertainties. Taking serious account of sex/gender - one of the most significant collective experiences of contemporary subjectivity - is fundamental to developing more promising responses to the growth in complex bio- 
medical knowledge and consequent uncertainties.

\section{Notes}

1 This history is well documented within feminist research in the area of health, but also more broadly. See for example, Graham (1984) and Gregory (2005). More broadly, this paper assumes that bodies are always sexed and can never be "neutral". For philosophical explanations of this position see, for example, Grosz (1994); Gatens (1996); Butler (1993). For discussions of the sexed body in twentieth century biomedicine and technoscience, see Hird (2004). For discussions of the importance of sex hormones to such understandings, see Oudshoorn (1994); Roberts (forthcoming).

2 Rose and Novas (2005: 447-8) discuss this connection in the case of pharmaceutical giant Eli Lilly's website for their drug, Prozac. Describing a website that encourages patients to ask doctors to prescribe Prozac, and explains depression solely in neurochemical terms, Rose and Novas write: "The role of biomedical authority here is not to encourage the passive and compliant patienthood of a previous form of medical citizenship. Citizenship, here as elsewhere, must be active. Thus the potential patient must try to understand his or her depression, to work with his or her doctor to obtain the best program of medical care, to engage in self-techniques to speed the process of recovery - and, of course, to ask his or her doctor to prescribe Prozac by name" (Rose and Novas, 2005: 448; emphasis added). Thus arguably, websites like this may encourage new forms of active patienthood that are also compliant with pharmaceutical treatment regimes that allow companies like Eli Lilly "to maintain market share" (Rose and Novas, 2005: 448. See also footnote 6 below).

3 A Lexis-Nexus search on the keywords "endocrine disruptors" brings up articles from all of these newspapers.
4 In her study of the management of diets related to chronic disease, Susan Gregory (2005) demonstrates the ways in which sex/gender relations are enacted through the provision and consumption of healthy meals in domestic settings.

5 There are also many self-help books that offer women advice and information about the menopause. Many of these follow a biomedical model, but mix in advice based on alternative therapies and psychology as well. They do not tend to include analysis of the social experience of aging, although there are some significant exceptions to this (Greer, 1991; Coney, 1991; Sheehy, 1993; Murray, 2001).

6 This figuration of responsibility for decisions about HRT-taking in individual cases is also evident in the medical literature addressed to clinicians (Roberts, 2002).

7 Newspaper articles both stimulated concern and tried to dampen it down. Wellknown medical figures such as Dr. Miriam Stoppard were cited to reinforce the benefits of HRT (Oliver, 2002). Early studies report a significant decrease in prescriptions of HRT in the United States after the termination of the clinical trial (Hersch, Steffanick and Stafford, 2004).

8 I use the term "enactment" in this paper to signal the ways in which subject positions, relations and material-discursive entities (such as "the menopause" or "femininity") are produced in particular times and spaces in interactions between humans and non-humans. This term has been suggested by science-studies theorist Annemarie Mol (2002: 32-3) in her recent book on ontology in medical practice.

9 Other authors analyse the ways in which gender is done in clinical encounters with male patients, see for example, Ferzacca (2000); Watson (2000).

10 Henwood et al. (2003: 591) cite DixonWoods who argues in her analysis of patient information leaflets, that such information might more honestly be called "information for compliance" than "infor- 
mation for choice". This is based on the fact that the information tends to be predominantly biomedical and to "adopt a rather one-way model of communication" (Henwood et al., 2003: 591).

11 In discussing the work of Rayna Rapp, for example, Rose and Novas miss the fact that Rapp (1999: 306) describes women as "moral pioneers", rather than, as they suggest "women and men" (Rose and Novas, 2005: 450). The conceptual and political significance of Rapp's empirical focus on women is lost in their analysis.

\section{References}

Atkins, L.

2004 "Throw out the bath water?" Chemical World supplement, The Guardian, May 8: 20-23.

Batt, S.

1996 Patient No More: The Politics of Breast Cancer. Melbourne: Spinifex Press.

Berkson, D. L.

2000 Hormone Deception: How Everyday Foods and Products Are Disrupting Your Hormones - And How to Protect Yourself and Your Family. ContempoBlair, T. rary Books: Chicago.

2004 "Foreword." P. 3 in Department of Health. Choosing Health: Making Healthier Choices Easier. Norwich:The Stationary Office.

Bix, A. S.

1997 "Diseases chasing money and power: Breast cancer and AIDS activism challenging authority." Journal of Policy Butler, J. History 9(1): 5-32.

1993 Bodies That Matter: On the Discursive Limits of "Sex". New York and London: Routledge.

Callon, M. \& Rabeharisoa, V.

2004 "Gino's lesson on humanity: Genetics, mutual entanglements and the sociologist"s role." Economy and Society 33(1): 1-27.
Cleary, P. D.

1999 "Paternalism or partnership? Patients have grown up and there's no going back." British Medical Journal 319 (18 September): 719-720.

Coney, S.

1991 The Menopause Industry: A Guide to Medicine's "Discovery" of the Midlife Woman. Melbourne: Spinifex Press.

Department of Health

2004 Choosing Health: Making Healthier Choices Easier. Norwich, United Kingdom: The Stationary Office.

Dreyfuss, R.C. \& Nelkin, D.

1992 "The jurisprudence of genetics." Vanderbilt Law Review 45(2): 313-348.

Duden, B.

1993 Disembodying Women: Perspectives on Pregnancy and the Unborn. Cambridge, Mass.: Harvard University Press.

Dumit, J.

2002 "Drugs for Life." Molecular Interventions 2(3): 124-127.

Elwyn, G., Edwards, A., Gwyn, R. \& Grol, R.

1999 "Towards a feasible model for shared decision making: Focus group study with general practitioners." British Medical Journal 319 (18 September): 753-756.

Epstein, S.

2003 "Sexualizing Governance and Medicalizing Identities: The emergence of 'state-centered' LGBT health politics in the United States." Sexualities 6(2): 131171.

1996 Impure Science: AIDS, Activism and the Politics of Knowledge. Berkeley, CA: University of California Press.

Ericson, R., Barry, D., \& Doyle, A.

2000 "The moral hazards of neoliberalism: Lessons from the private insurance industry." Economy and Society 29(4): 532-58.

Ferzacca, S.

2000 “'Actually, I don't feel that bad': Managing diabetes and the clinical encounter." Medical Anthropology Quarterly 14(1): 28-50.

Foucault, M.

1985 The Use of Pleasure: The History of Sexuality, Vol. II. New York: Pantheon.

1987 [1976] The History of Sexuality: An introduction. London: Penguin Books. 
Friends of the Earth and the National

Childbirth Trust

Undated "Chemicals in the Home: A Parent's Guide." Safer Chemicals Campaign, Friends of the Earth: London.

Gatens, M.

1996 Imaginary Bodies: Ethics, Embodiment and Corporeality. New York and London: Routledge.

Graham, H.

1984 Women, Health and the Family. Brighton: Harvester Wheatsheaf.

Greenpeace

2003 Human Impacts of Man-made Chemicals. London: Greenpeace. www. greenpeace.org.uk/toxics. Last accessed 25 October 2006.

2005 Poisoning the Unborn. www. greenpeace.org/international/mews/ poisoning-the-unborn111. Last accessed 26 October 2006.

Greer, G.

1991 The Change: Women, Aging and the Menopause. London: Hamish Hamilton Ltd.

Gregory, S.

2005 "Living with chronic illness in the family setting." Sociology of Health and Illness 27(3): 372-92.

Griffiths, F.

1999 "Women's control and choice regarding HRT." Social Science and Medicine 49: 469-81.

Griffiths, F., Green, E. \& Tsouroufli, M.

2005 "The nature of medical evidence and its inherent uncertainty for the clinical consultation: Qualitative study." British Medical Journal 330: 511-8.

Griffiths, F., Green, E. \& Bendelow, G.

2006 "Health professionals, their medical interventions and uncertainty: A study focusing on women at midlife." Social Science and Medicine 62: 1078-90.

Grosz, E.

1994 Volatile Bodies: Toward a Corporeal Feminism. Sydney: Allen and Unwin.

Guillemin, M.

2000a "Blood, bone, women and HRT: Coconstruction in the menopause clinic." Australian Feminist Studies 15(32): 191-203. 2000b "Working practices of the menopause clinic." Science, Technology and Human Values 25(4): 448-470.

Health Promotion England

2000 The Menopause: The Facts about the Menopause, HRT and Osteoporosis. London: National Health Service.

Helén, I.

2004 "Technics over life: Risk, ethics and the existential condition in high-tech antenatal care." Economy and Society 33(1) February: 28-51.

Henwood, F., Wyatt, S., Hart, A. \& Smith, J.

2003 “'Ignorance is bliss sometimes': Constraints on the emergence of the 'informed patient' in the changing landscapes of health information." Sociology of Health and Illness 25(6): 589-607.

Hersh, A. L., Stefanick, M. L., Stafford, R. S.

2004 "National use of postmenopausal hormone therapy: Annual trends and response to recent evidence." Journal of the American Medical Association 291:47-53.

Hird, M. J.

2004 Sex, Gender and Science. Hampshire and New York: Palgrave Macmillan.

Hoffman, M., Lindh-Åstran, L., Ahlner, J.,

Hammar, M. \& Kjellgren, K. I.

2005 "Hormone replacement therapy in the menopause: Structure and content of risk talk." Maturitas 50(1): 8-15.

Hunter, M. S., O’Dea, I. \& Britten, N.

1997 "Decision-making and hormone replacement therapy: A qualitative analysis." Social Science and Medicine 45(10): 1541-1548.

Krimsky, S.

2000 Hormonal Chaos: The Scientific and Social Origins of the Environmental Endocrine Hypothesis. Baltimore and London: Johns Hopkins University Press.

Lawrence, $\mathrm{F}$.

2004 "Bugs, sweat and fears." The Guardian Weekend supplement, May 12: 14-22.

Locock, L. \& Alexander, J.

2006 “'Just a bystander?' Men's place in the process of fetal screening and diagnosis." Social Science and Medicine 62(6): 1349-1359. 
Lippman, A.

1992 "Led (astray) by genetic maps: The cartography of the human genome and health care." Social Science and Medicine 35(12): 1469-76.

Lyons, A. C. \& Griffin, C.

2003 "Managing menopause: A qualitative analysis of self-help literature for women at midlife." Social Science and Medicine 56: 1629-1642.

Massé, R., Legare, F., Cote, L. \& Dodin, S.

2001 "The limitations of a negotiation model for perimenopausal women." Sociol$\mathrm{Mol}, \mathrm{A}$. ogy of Health and Illness 23(1): 44-64.

2002 The Body Multiple: Ontology in Medical Practice. Durham and London: Duke University Press.

Murtagh, M. J. \& Hepworth, J.

2003a "Menopause as a long-term risk to health: Implications of general practitioner accounts of prevention for women's choice and decision-making." Sociology of Health and Illness 25(2): 185207.

2003b "Feminist ethics and menopause: $\mathrm{Au}-$ tonomy and decision-making in primary medical care." Social Science and Medicine 5: 1643-1652.

Murray, J.

2001 Is it Me, or is it Hot in Here? A Modern Woman's Guide to the Menopause. London: Vermillion.

National Institutes of Health.

2002 "NHLBI stops trial of estrogen plus progestin due to increased breast cancer risk, lack of overall benefit." NIH News Release, Tuesday July 9. www. nhlbi.nih.gov/new/press/02-0709.htm.

Novas, C. \& Rose, N.

2000 "Genetic risk and the birth of the somatic individual." Economy and Society 29(4): 485-513.

NovoNordisk Ltd.

(undated) Choosing HRT: Weighing up the facts $\mathrm{KV} / 01 / 72$.

Oliver, $\mathrm{M}$.

2002 "HRT may be more risky than thought." The Guardian Unlimited, Wednesday July 10. www.guardian.co.uk.
O'Malley, P.

2000 "Uncertain subjects: Risks, liberalism and contract." Economy and Society 29(4): 460-484.

Oudshoorn, N.

1994 Beyond the Natural Body: An Archeology of Sex Hormones. London and New York: Routledge.

Rabinow, P.

1992 "Artificiality and enlightenment: From sociobiology to biosociality." Pp. 234252 in Crary and Kwinter (eds.), Incorporations. New York: Zone Books.

Rapp, R.

1999 Testing Women, Testing the Fetus: The Social Impact of Amniocentesis in America. New York: Routledge.

Rapp, R., Heath, D. \& Taussig, K-S.

2001 "Genealogical dis-ease: Where hereditary abnormality, biomedical explanation, and family responsibility meet." Pp. 384-411 in Franklin and McKinnon (eds.), Relative Values: Reconfiguring Kinship Studies. Durham, NC: Duke University Press.

Roberts, C.

2002 “'Successful aging' with hormone replacement therapy: It may be sexist, but what if it works?" Science as Culture 11(1): 39-59.

2003 "Sex, race and 'unnatural' difference: Tracking the chiastic logic of menopause-related discourses." European Journal of Women's Studies 11(1): 2744.

2006 "Enacting gender in reproductive medicine.” Pp. 87-100 in Wieser, Karner \& Berger (eds.), Prenatal Testing: Individual Decision or Distributed Action? München, Wien: Profil.

Forthcoming Messengers of Sex: Hormones, biomedicine and feminism. Cambridge: Cambridge University Press.

Roberts, C. \& Franklin, S.

2005 "Experiencing new forms of genetic choice: Findings from an ethnographic study of preimplantation genetic diagnosis." Human Fertility 7(4): 285-294. 
Rose, N. \& Novas, C.

2005 “Biological citizenship." Pp. 439-463 in Ong and Collier (eds.), Global Assemblages: Technology, Politics, and Ethics as Anthropological Problems. Malden, Oxford and Carlton: Blackwell.

Samerski, S.

2005 "Autonomy and Therapy." New Perspectives Quarterly 22(10): 71-77.

Sharpe, R. M. \& Irvine, D. S.

2004 "How strong is the evidence of a link between environmental chemicals and adverse effects on human reproductive health?" British Medical Journal 328 (21 February): 447-451.

Sheehy, G.

1993 Menopause: The Silent Passage. London: Harper Collin.

Stacey, J.

1997 Teratologies: A Cultural Study of Cancer. London and New York: Routledge.

2000 "The Global Within: Consuming nature, embodying health." Pp. 97-145 in Franklin, Lury \& Stacey, Global Nature, Global Culture. London: Sage Publications.

Tanenbaum, S. J.

2005 "Commentary: Uncertainty, consultation, and the context of medical care." British Medical Journal 330: 515.

Thompson, C.

2005 Making Parents: The Ontological Choreography of Reproductive Technologies. Cambridge, Mass.: The MIT Press.

Throsby, K.

2004 When IVF Fails: Feminism, Infertility and the Negotiation of Normality. Houndsmills and New York: Palgrave Macmillan.

Throsby, K. \& Gill, R.

2004 “'It's different for men': Masculinity and IVF." Men and Masculinities 6(4): 330348.

Watson, J.

2000 Male Bodies: Health, Culture and Identity. Open University Press: Buckingham and Philadelphia.

Wieser, B., Karner, S. \& Berger, W.

2005 "Who is responsible for distributed action? On the paradoxes of prenatal testing." Pp. 1-14 in Berger (ed.), Prenatal Testing: Individual Decision or Distributed Action? Interim Project Report,
Institute for Interdisciplinary Studies. Department of Research on Science and Technology: Klagenfurt.

World Wildlife Fund

2002 "Who Cares Where Toxic Chemicals End Up?" [Awareness-raising campaign]. Country Living, February: 43; Observer Food Monthly, February: 25. www.wwf.org.uk/whocares/page3.asp.

2006 "WWF Toxics Programme". www. panda.org/about_wwf/what_we_do/ toxics/index.cfm. Last accessed 23 October 2006.

Writing Group for the Women's Health Initiative Randomized Controlled Trial 2002 "Risks and benefits of estrogen plus progestin in healthy post-menopausal women." Journal of the American Medical Association 288(3): 321-323.

\section{Celia Roberts \\ Department of Sociology \\ University of Lancaster, UK \\ celia.roberts@lancaster.ac.uk}

